black smoke is given off, and the compound is completely decomposed. On quinine iodate the strongest sulphuric acid produces merely a change from a white to a very light yellow colour; and the addition of water formas a colourless solution. At $100^{\circ}$ Centigrade, quinine iodate undergoes slight decomposition.

A brief reference to an iodate of quinine is made by Serullas, but of bromate of quinine no account could be fouud in the books or journal. It may be prepared by precipitating barium bromate by sulphate of quinine, and evaporating the solution separated from the barium sulphate, also by neutralising bromic acid solution with quinine. It occurs in silky minute needles, very soluble in water, spirit of wine, and dilute acids, except nitric acid. It is fully as active in its physiological effects as the iodate. It may be prescribed as a mixture, in pills, or in the granular effervescing mixture of the Pharmacopoeia. It is soluble in 250 parts of water. Soon after the administration of the iodate of quinine, iodic and hydriodic acids appear in the urine. The quinine also appears, but arrives somewhat later.

\section{DORSAL DISLOCATION OF THE LEFT HIP (FOUR MONTHS); REDUCTION BY MANIPULATION.}

BY THOMAS JONES, F.R.C.S.,

SURGEON TO THE MANCHESTER ROYAL INEIRMARY.

INSTANCES of the successful replacement of the head of the femur after its sojourn for several months on the dorsum ilii are sufficiently rare to justify the narration of a case. Although Sir A. Cooper's dictum (which in the case of the hip fixes the period at two months, beyond which attempts at reduction are impracticable) is generally adhered to, still examples are not wanting in which reduction has been effected after the lapse of a much longer period. Thus, Bigelow cites cases where reduction was accomplished six and eight months after the accident. It is true the difficulties inseparably associated with the efforts to reduce old dislocations are very great and often insurmountable, and the attendant risks always considerable, yet the attempt is rendered necessary because the utility of a $\operatorname{limb}$ is seriously impaired by the presence of the unreduced bone. In the case which is narrated below the restoration of function after the replacement of the femur was fairly good. This shows that a complete separation of the joint surfaces for many weeks is not necessarily followed by much structural change. The free mobility of the head made it tolerably certain that the ligamentum teres was severed, and the capsule freely torn-an amount of destruction of soft parts which we should expect to be followed by, at any rate, some deformity of the head. The ease with which the head regained the acetabulum was a source of surprise and satisfaction. The movements presently to be mentioned sufficed to bring the displaced bone into its natural situation, and this occurred without the employment of any force. When the limb was flexed at the knee, and rotated in wards, the head readily escaped from the acetabulum, but was again replaced by movements similar to those employed in the first instance. This ready escape of the head may be taken as an indication of, possibly, two conditions: the free severance of soft structures, and the partial obliteration of the cup shaped acetabulum. That this latter condition really existed is highly probable, and is rendered almost certain by the lengthening of the limb, which was found after the reduction. It is important to note the position of the patient immediately before the accident. $\mathrm{He}$ tells us he was stooping; that his legs were apart (in a position, then, of abduction of the limbs, and flexion of the trunk on the thighs), and that while in this state he received the blow which occasioned the dislocation. This will tend to confirm Mr. Morris's observation respecting the mode in which dorsal dislocations are produced. The history of the case may now be related :-

Wm. H- aged twenty-four, was admitted an in-patient of the Infirmary on the 27th January, 1882, and furnished the following history. On the 8th of October, 1881, he was playing at fuotball, and whilst stooping with his legs apart he received from behind a severe push which threw him to the ground. On attempting to stand the left leg was diseovered to be very much inverted, and a total inability to walk immediately followed. An irregular practirioner in the shape of a "horse doctor" manipulated the injured limb, and informed the patient that the dislocation had been reduced.

Condition on edmission.-The left lower extremity is strongly inverted and shortened to the extent of two inches. It permits of rotation inwards, and inversion; but rotation outwards and eversion are impossible. The trochanter major is very prominent and nearer to the anterior superior spine of ilium than that on the other side. The head is easily discovered on the dorsum ilii, where it is very superficial and freely movable. Syme: distinctive sign of dorsal dislocation is absent, for the thigh can be made to touch the bed without any arching of the back.

Jan. 30th. - The patient was anresthetised, and the head of the femur returned into the acetabulum by the following movements. The ankle of the dislocated limb was grasped with the right hand, while the left rested on the knee. Then the thigh was flexed upon the abdomen, and the limb rotated inwards to a slight degree. This was done with the object of more effectually disentangling the head. Immediately afterwards the limb was abducted, rotated outwards and extended, when the head regained the acetabulum with a sound which was audible to the students present. It was now discovered that the inversion and the other symptoms had completely disappeared, and that a quarter of an inch lengthening was substitnted for the previous shortening. Redisplacement could be readily made by flexing the limb and rotating it inwards. To remove any chance of the dislocation recurring a Liston's splint was applied.

Feb. 6th.-Splint temporarily removed; a certain amount of motion in the hip-joint discovered; splint reapplied.14th : Felt moulded to the hip, and the $\operatorname{limb}$ encased in a plaster-of-Paris bandage carried upwards from the foot. Hip-joint made secure by a pelvic spica,-16th : Patient discharged.

April 11th.-Readmission. Plaster bandage taken off. Hip and knee-joints found to be stiff. Leo lengthened and somewhat abducted.-14th : Patient again placed under the influence of chloroform with the view of breaking down adhesions. The different movements naturally enjoyed by the hip can be performed by the expenditure of some degree of force. Both the hip and knee to be freely rubbed with soap liniment and passive movements to be employed twice a day. By these means motion in the hip-joint was still further improved, so that by the time the patient was finally discharged, on April 18th, the usefuiness of the limb was almost completely restored, and very little inconvenience in walking experienced.

\section{A NEW MEDICAL ELECTRODE.}

\section{BY A. HUGHES BENNETT, M.D.}

PHYSICIAN TO THE HOSPITAL FOR EPILEPSY AND PARALYSIS, REGENT'S PARK, AND ASSISTANT-PHYSICIAN TO THE WESTMINSTER HOSPITAL.

IN the scientific employment of electricity in medicine, and more especially for the purposes of electro-diagnosis, in addition to the currents from different batteries, certain accessories are essential. There must be : lst. An electrode of suitable size and shape. 2nd. A method of graduating the strength of the current, by means of which the feeblest or the most powerful efforts of the battery may be obtained, or any intermediate grade that may be desired; this, moreover, must be effeeted gradually so as to avoid unnecessary sudden changes or shock. 3rd. A means of opening and closing or of interrupting the current. 4th. A mode of alternating or reversing the direction of the current.

Many of the batteries supplied for medical purposes do not possess all these accessories, which are absolutely essential for scientific investigation. Even those which supply these necessaries are open to the objection that in delicate diagnostic investigations the attention of the observer is divided between the electrode with which he makes his experiments on the patient and the element-board upon 
which are placed the different apparatus he has to manipulate. To those practically experienced in electro-diaynostic

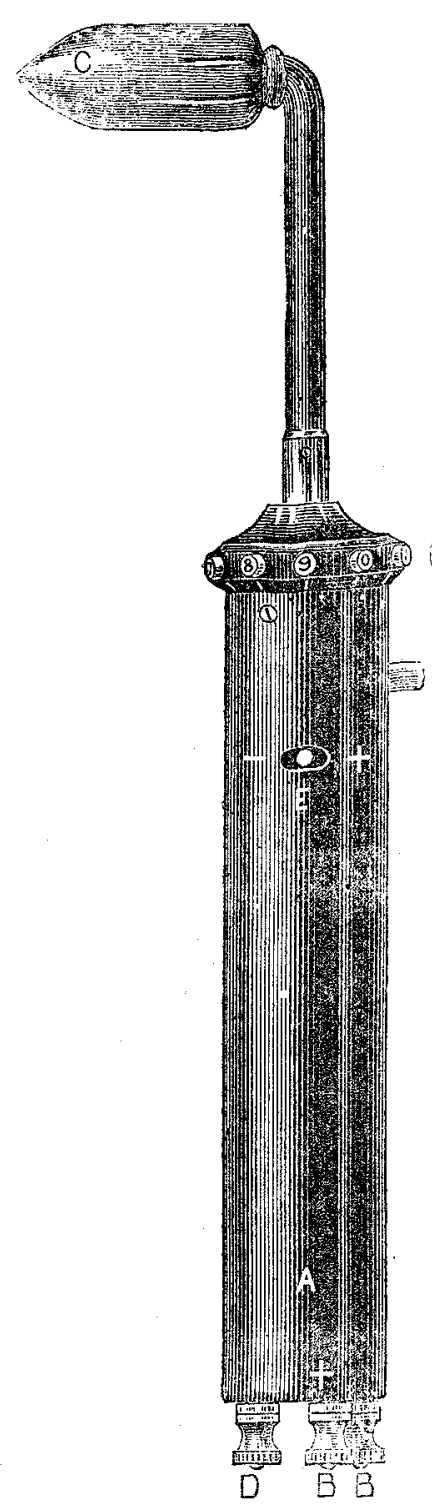
researches this has always been found to be a serious drawback to the facility and success of their conclusions. In the endeavour to in some measure obviate these difficulties the instrument has been constructed of which the accompanying woodcut (half size) indicates the chief features. It consists of an electrode of convenient shape and size, and contains in its interior all the essential accessories above enumerated. $A$ is the handle. B B are two connecting screws, to which are attached the positive and negative poles of the battery. $C$ is a metal or carbon point, covered with wash-leather; in the figure this is of a convenient shape for electro-diagnostic purposes, by which either a very fine point or a considerable surface can be applied to the skin; it may be unscrewed, and other heads of different shapes and sizes fixed on as necessity may require. $D$ is a binding screw for the other electrode, which is applied to the patient in order to complete the circuit. $\mathbf{E}$ is a lever for alternating the cur. rent. When moved towards + the point $C$ is the positive, and when towards - it is the negative, pole. $F$ is a peg which when pressed down closes, and when it moves back by means of a spring breaks the circuit; the current can thereby be interrupted as slowly or as quickly, up to a certain point, as is desired. $G$ is a revolving wheel in connexion with a rheostat in its interior. This consists of ten sections, numbered respectively; and each of these, by throwing in a unit of resistance, in consequence diminishes the strength of the current, and vice versa. This rheostat does not profess to be an exact or measurable amount of resistance, but is simply a means of gradually increasing or diminishing the strength of the current for practical purposes. It has been so arranged that its power is convenient for clinical use. For example, when the instrument is attached to the full power of a forty-cell Leclanché battery, and the entire resistance is introduced into the circuit, a minimum amount of sensation is perceptible to the skin. As each division of the wheel is turned a tenth of the whole resistance is taken from the circuit, and consequently the current increases in strength, till when it reaches the last section, where there is no resistance, the full power of the forty cells is obtained. More correctly estimated by a galvanometer, the properties of this rheostat may be thus stated: Supposing the current from the forty cells passing through the human body to indicate $12 \mathrm{~m} . \mathrm{v}$, one unit of resistance of the rheostat introduced into the circuit reduces the reading to $11 \mathrm{~m}$.v., and so on, till when the whole resistance is included $2 \mathrm{~m} . \mathrm{v}$. is the result.

This electrode therefore contains in itself all the essential elements for medical electrical appliances, and more especially for the purposes of electro-diagnosis. It mav be attached to either the faradaic or galvanic currents. When in the hand of the observer, all the accessories can be put in action by the movement of one finger, thus greatly facilitating the delicate manipulations of the investigator, and obviating the necessity of dividing his attention and altering his position, as he is compelled to do when working with an element-board. Finally, with this instrument, which can be carried in the pocket, the physician, provided he obtains a sufficiently powerful current, is independent of the imperfect construction of most of the apparatus now supplied to the profession, as all the necessary accessories be requires are contained in his electrode. The instrument has betn manufactured by Mr. Hawksley, Oxford-street. Old Carendish.street, $w$.

\section{A CLINICAL STUDY OF THE}

\section{SMALL GRANULAR CELLS OF THE BLOOD.}

BY JAMES T. R. DAVISON, M.D. EDIN,

LATE RESIDENT PHYSICIAN TO THE ROYAL INFIRMARY, EDINBURGH; HOUSE-SURGEON TO THE ROYAL SOUTHERN HOSPITAL,

$$
\text { LIVERPOOL. }
$$

BLOod contains, in addition to the red and white corpuscles, a number of granular colourless cells, which present varied forms and sizes, and are generally very much smaller than the red corpuscles when examined in the ordinary way. They have a great tendency to adhere to the cover-glass or slide; they occur singly or in groups. Some time after the blood has been drawn from the finger these cells become clearer; if present in groups the individuality of the cells is lost, and the group becomes a single granular mass. In either case fibrin or fibrin-like prosesses protrude from their surface. These cells have been known for some time to Professor Norris, who has traced them in their development, and states them to be undeveloped red corpuscles. Bizzozero has lately studied them in the living capillary. In healthy blood they are present in extremely small numbers, but are very abundant in certain pathological states. I have examined these cells in 150 specimens of pathological blood, noting in each case the temperature of the patient from whom the blood was drawn, whether anæmic or not (in the last 100 cases, if anæmic, I noted rougbly the degree of anæmia present), and lastly the nature and duration of the disease.

My observations lead me to believe that the small granular cells of the blood are present in excess under two conditions-firstly, as the result of their excessive formation brought about by certain states of the system demanding it; and secondly, as the result of diminishted power in the process of charging them with hromoglobin, whereby many of them remain in their undeveloped state, and do not become fully formed red cells.

In the inflammatory exudation produced by a blister, Dr. Burdon-Sanderson years ago found granular corpuscles which degenerated into fibrin. These corpuscles must be the cells under consideration at present, for these cells are constantly seen to change into fibrin, while leucocytes never undergo this change. Here, then, we have the important fact that in inflammatory serous exudation, such as we have in pleurisy, rheumatic fever, \&c., the small granular corpuscles of the blood migrate from the bloodvessels. Dr. Burdon-Sanderson in his first Lumleian lecture this year described ordinary inflammation of the mesentery, and after referring to the migration of lencocytes he states that the surface of the mesentery becomes covered by a coagulable fluid. This coagulum, when examined under the microscope, is seen to consist of fibrin which Dr. Burdon-Sanderson ascribes to the degeneration of leucocytes. Now, if a drop of blood be examined under the microscope the leucocytes are seen to remain intact for a long time, and never undergo the same changes as the small granular cells-that is, they never degenerate into fibrin. Moreover, Professor Norris has found that the fibrin of coagulation is due to changes in the undeveloped red cells. We must come to the conclusion, then, that the coagulable fluid on the surface of the mesentery was full of undeveloped red cells, which had degenerated into fibrin-that is, that in ordinary inflamma. tion, in addition to the migration of leucocytes, there is a migration of the small granular corpuscles. We have good grounds, then, for believing that not only in serous inflammatory exudation, but also in purulent exudation, there is a migration of the undeveloped red corpuscles. If, then, in local inflammation the blood is drained of its small granular corpuscles, nature must in some reflex manner stimulate the formative organs of these cells to increased action, in order to meet the demand made upon the blood. Hence in local inflammations we would expect to find an increase of these cells in the blood. I will not refer in these notes to the part played by leucocytes in the blood 